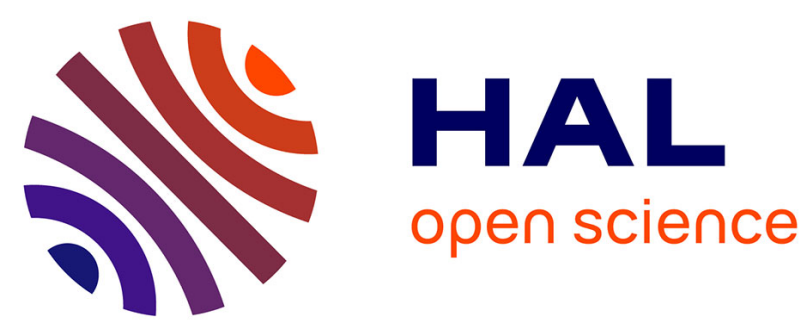

\title{
Anti-phase wave patterns in a ring of electrically coupled oscillatory neurons
}

Aleksei S. Dmitrichev, Vladimir I. Nekorkin, Rachid Behdad, Stéphane Binczak, Jean-Marie Bilbault

\section{To cite this version:}

Aleksei S. Dmitrichev, Vladimir I. Nekorkin, Rachid Behdad, Stéphane Binczak, Jean-Marie Bilbault. Anti-phase wave patterns in a ring of electrically coupled oscillatory neurons. The European Physical Journal. Special Topics, 2013, 222, pp.2633-2646. 10.1140/epjst/e2013-02043-6 . hal-00909693

\section{HAL Id: hal-00909693 https://hal.science/hal-00909693}

Submitted on 26 Nov 2013

HAL is a multi-disciplinary open access archive for the deposit and dissemination of scientific research documents, whether they are published or not. The documents may come from teaching and research institutions in France or abroad, or from public or private research centers.
L'archive ouverte pluridisciplinaire HAL, est destinée au dépôt et à la diffusion de documents scientifiques de niveau recherche, publiés ou non, émanant des établissements d'enseignement et de recherche français ou étrangers, des laboratoires publics ou privés. 


\title{
Anti-phase wave patterns in a ring of electrically coupled oscillatory neurons
}

\author{
Alexey S. Dmitrichev ${ }^{1, a}$, Vladimir I. Nekorkin ${ }^{1}$, Rachid Behdad ${ }^{2}$, Stephane \\ Binczak $^{2}$, and Jean-Marie Bilbault ${ }^{2}$ \\ 1 Institute of Applied Physics of RAS, 46 Uljanov str., 603950 Nizhny Novgorod, Russia \\ 2 LE2I CNRS UMR6306, University of Burgundy, BP47870, 21078 Dijon, France
}

\begin{abstract}
Space-time dynamics of the network system modeling collective behavior of electrically coupled nonlinear cells is investigated. The dynamics of a local cell is described by the dimensionless MorrisLecar system. It is shown that such a system yields a special class of localized collective activity so called "anti-phase wave patterns". The mechanisms of formation of the patterns are discussed. By using the weakly coupled oscillators theory the region of their existence is obtained.
\end{abstract}

\section{Introduction}

Having a clear view of the neuronal system dynamics is significant for an understanding of their functions and it is also irreplaceable when one endeavors to devise mathematical models of the neurons or neuronal networks. The most popular and practically important branch in such kind of researches is an investigation of small ensembles of neurons, where both neurons and their mutual coupling are described by sufficiently realistic mathematical models, based on the experimental data [1]. But in spite of simplicity of such models, they possess a great number of collective behavior regimes on account of ultrahigh multistability. Certainly, some of these regimes have no definite physical interpretation, but nevertheless, it can be of service to understand the basic information transmission processes in the brain.

The functioning of such complex neuronal systems as retina and other sensory networks is one of the crucial problems of neuroscience nowadays [2-4]. Retinal structure is adequately high-ordered, which would have simplified to devise mathematical models and to simulate the networks numerically. But at the same time this results in general view of connectivity patterns and brain principles of operation at large. At present the new mathematical models of retinal-like structures are suggested and some progress in understanding basic properties of such systems is achieved. But we take the view, that the very neuron models mentioned above can be useful in investigating the ordered neuronal structures.

In Fig. 1 the most typical impulse responses of retinal ganglion cells are shown. It is important that the surround cell response is out of phase with the central one. This makes possible to apply an anti-phase synchronization model of two electric-coupled

\footnotetext{
a e-mail: admitry@neuron.appl.sci-nnov.ru
} 

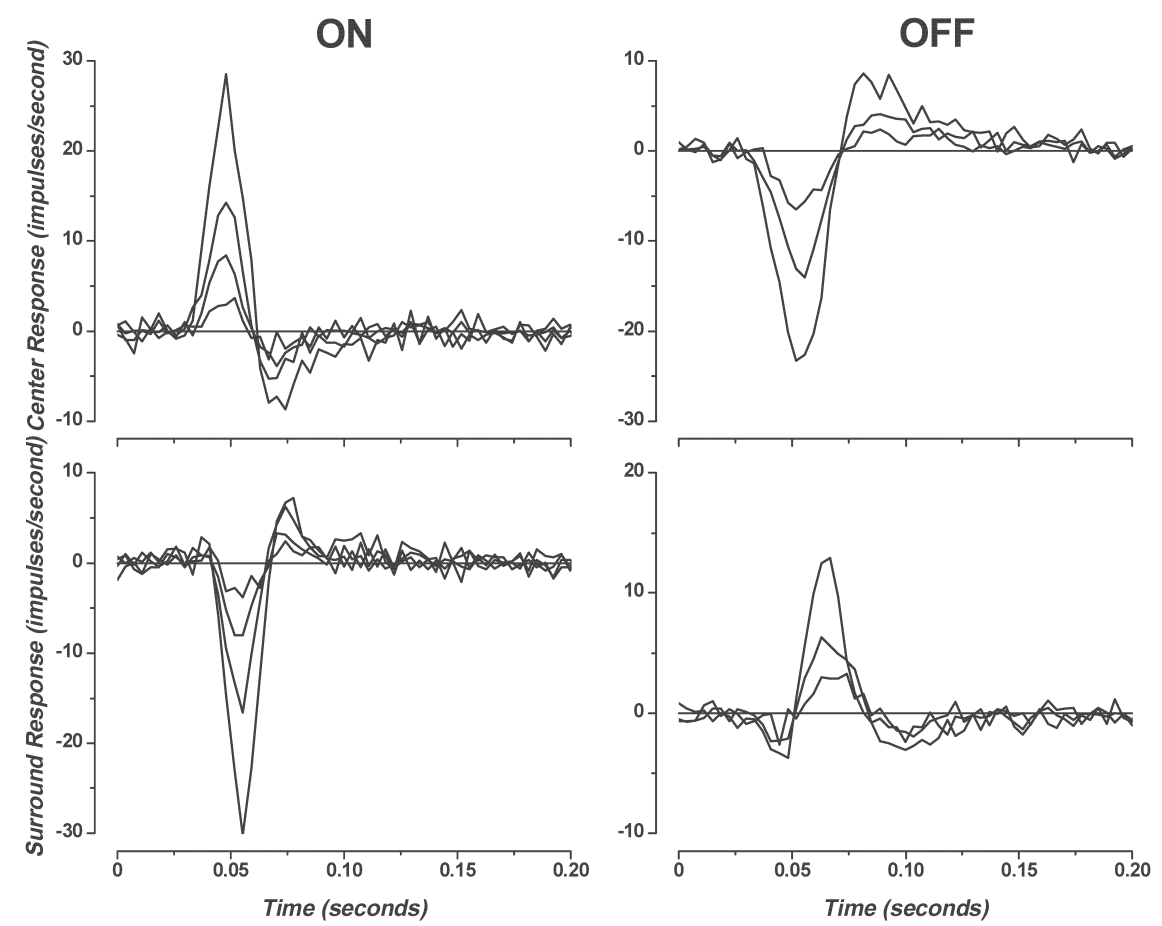

Fig. 1. The first order responses of a $\mathrm{P}$-type primate ganglion cell center and surround, adopted from [2].

neurons to account for the fact of such complicated oscillatory structures existence that will be discussed later.

The paper is organized as follows. In Section 2 we describe the model studied and briefly discuss its basic dynamical properties. A special class of localized collective activity obtained in the model which was called "anti-phase wave patterns" is presented in Section 3. We show that the formation of anti-phase wave patterns is related to establishing of a certain phase relations between oscillations of neighboring elements. In Section 4 we use weakly coupled oscillators theory to study the regions of the existence of such patterns. Finally, the main conclusions are summarized in Section 5 .

\section{The dynamical model}

We consider the following network composed of identical neural-like units or cells arranged on a one-dimensional ring and coupled locally in a nearest-neighbor fashion:

$$
\begin{aligned}
& \left\{\begin{array}{l}
\frac{d \bar{v}_{i}}{d t}=-\bar{g}_{L}\left(\bar{v}_{i}-\bar{v}_{L}\right)-\bar{g}_{C a} M_{\infty}\left(\bar{v}_{i}\right)\left(\bar{v}_{i}-\bar{v}_{C a}\right)-\ldots \\
\ldots-\bar{g}_{K} n_{i}\left(\bar{v}_{i}-\bar{v}_{K}\right)+\bar{I}+d\left(\bar{v}_{j-1}-2 \bar{v}_{j}+\bar{v}_{j+1}\right) \\
\frac{d n_{i}}{d t}=\varepsilon \frac{n_{\infty}\left(\bar{v}_{i}\right)-n_{i}}{\tau_{n}\left(\bar{v}_{i}\right)}
\end{array}\right. \\
& i=1,2, \ldots N, \quad \bar{v}_{j+N}(t) \equiv \bar{v}_{j}(t) .
\end{aligned}
$$

The variable $\bar{v}_{i}$ describes the evolution of the membrane potential of a neuron located at the $j$ st site of the ring, $n_{i}$ describes the dynamics of the activation level of the 
potassium ion channels. The terms on the right-hand side of the first equation of (1) accounts for the currents flowing through the cell membrane of the neuron. The first three terms correspond to the leakage, the calcium and the potassium ionic currents, respectively. The last ones signify the external (applied) and the electrical synaptic currents. The parameters $\bar{g}_{L}, \bar{g}_{C a}$, and $\bar{g}_{K}$ are the maximum conductances, $\bar{v}_{L}, \bar{v}_{C a}, \bar{v}_{K}$ are the equilibrium potentials (reverse potentials) for the corresponding ion channels. The functions $M_{\infty}(\bar{v})$ and $n_{\infty}(\bar{v})$ obeying the following laws:

$$
\begin{aligned}
& M_{\infty}(\bar{v})=0.5\left[1+\tanh \left(\frac{\bar{v}-\bar{v}_{1}}{\bar{v}_{2}}\right)\right], \\
& n_{\infty}(\bar{v})=0.5\left[1+\tanh \left(\frac{\bar{v}-\bar{v}_{3}}{\bar{v}_{4}}\right)\right]
\end{aligned}
$$

define, respectively, the stationary activation levels for calcium and potassium ion channels. Function $\tau_{n}(\bar{v})$ which obeys the law:

$$
\tau_{n}(\bar{v})=\left[\cosh \left(\frac{\bar{v}-\bar{v}_{3}}{2 \bar{v}_{4}}\right)\right]^{-1}
$$

and parameter $\varepsilon(\varepsilon>0)$ both control the characteristic relaxation time for potassium ion channels. Finally the parameter $d$ characterizes the strength of electrical inter-neuron coupling. The action of electrical coupling is based on the existence of so-called gap junction between neighboring neurons [5]. Such junction leads to direct connection of the cytoplasm of the neurons and allows various molecules and ions to pass freely between them. Hereupon the current passing through the gap junction obeys Ohm's law and depends directly on gap conduction (or inversely on gap resistance) and directly on difference in electric potential between neurons. Thus the electrical coupling is well-approximated by linear resistive coupling. Therefore in (1) the coupling terms are expressed in the form of standard one-dimensional discrete Laplacian.

\subsection{Local cell dynamics}

Let us first consider the dynamics of a single unit. It is described by the dimensionless version of the well known Morris-Lecar system [6]:

$$
\left\{\begin{array}{l}
\frac{d \bar{v}}{d t}=-\bar{g}_{L}\left(\bar{v}-\bar{v}_{L}\right)-\bar{g}_{C a} M_{\infty}(\bar{v})\left(\bar{v}-\bar{v}_{C a}\right)-\bar{g}_{K} \bar{n}\left(\bar{v}-\bar{v}_{K}\right)+\bar{I} \\
\frac{d n}{d t}=\varepsilon \frac{n_{\infty}(\bar{v})-n}{\tau_{n}(\bar{v})}
\end{array}\right.
$$

To derive it from the original system we rescaled the time properly and used the following transformations of variables and parameters:

$$
\begin{aligned}
& v=\frac{\bar{v}}{v_{0}}, v_{m}=\frac{\bar{v}_{m}}{v_{0}}, g_{m}=\frac{\bar{g}_{m}}{g_{0}}, v_{i}=\frac{\bar{v}_{i}}{v_{0}}, v_{0}=\frac{I}{\bar{I} g_{0}}, g_{0}=\frac{\phi C}{\varepsilon} \\
& m=L, C a, K, \quad i=\overline{1,4}
\end{aligned}
$$

where $C$ is the membrane capacitance and $\phi$ is the maximum relaxation time for potassium ion channels.

The original Morris-Lecar system is known to provide a wealth of dynamical behaviours. Detailed bifurcation analysis of its dynamics can be found, for example, in [7]. Here we briefly discuss only the dynamical regimes essential for the formation of anti-phase wave patterns. For our purposes we fix the parameters:

$$
\begin{aligned}
& \bar{g}_{L}=2, \bar{v}_{L}=-60, \bar{g}_{C a}=4, \bar{v}_{C a}=120, \bar{g}_{K}=8, \bar{v}_{K}=-80, \\
& \bar{v}_{1}=-1.2, \bar{v}_{2}=18, \bar{v}_{3}=12, \bar{v}_{4}=17.4,
\end{aligned}
$$




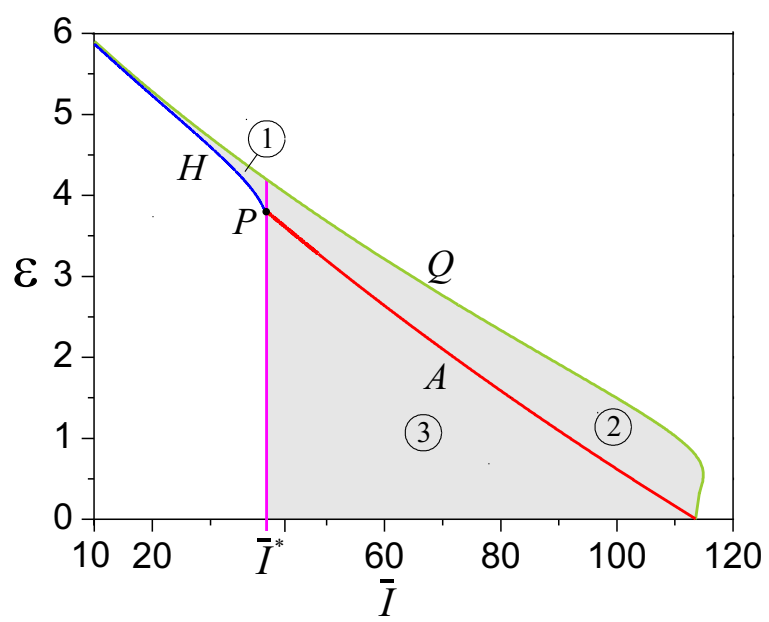

Fig. 2. Partition of a part of $(\varepsilon, \bar{I})$-plane on regions corresponding to different dynamical regimes of the system (4) for parameters given by (6), where $\bar{I}^{*}=39.69$ and $P(3.8,39.69)$ is intersection point of the curve $H$, and line $\bar{I}=\bar{I}^{*}$.

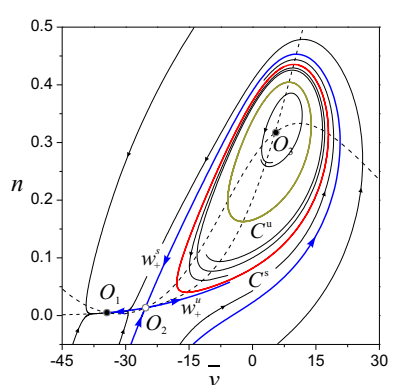

(a)

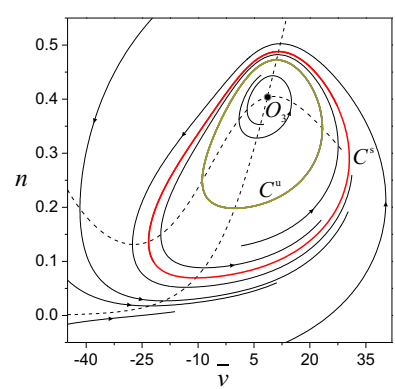

(b)

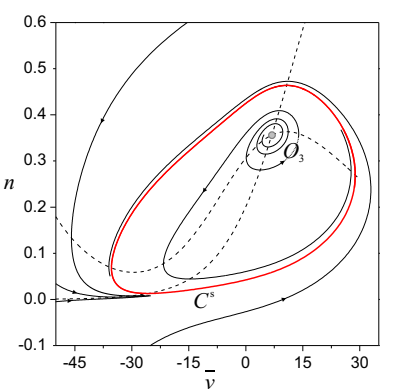

(c)

Fig. 3. Structurally stable phase portraits of system (4) for parameters given from the region $1: \varepsilon=4.2, \bar{I}=38(\mathrm{a})$; the region $2: \varepsilon=1.8, \bar{I}=90(\mathrm{~b})$; and the region $3: \varepsilon=2, \bar{I}=60$ (c); of the diagram in Fig. 2.

and take $\varepsilon$ and $\bar{I}$ as a control parameters. Figure 2 illustrates the partition of a part of $(\varepsilon, \bar{I}$ )-plane interested (highlighted by shaded area) onto regions with different dynamical behaviour of the system (4). According to the partition the system yields three different dynamical regimes which are depicted in its phase planes in Fig. 3 .

The main features of the regimes are existence of stable limit cycle $C^{s}$ (see Fig. 3) which corresponds to periodic oscillations of the membrane potential of a neuron. Depending on the value of parameter $\bar{I}$ there are also either one, or three steady states. Respectively, for $\bar{I}<\bar{I}^{*}$, that is in the region 1 (see Fig. 2), three steady states, $\mathrm{O}_{1}, \mathrm{O}_{2}$ and $\mathrm{O}_{3}$, exist (see Fig. 3(a)). The steady state $\mathrm{O}_{2}$ is of saddle type while the states $O_{1}$ and $O_{3}$ are either stable foci or nodes. For $\bar{I}=\bar{I}^{*}$ steady states $O_{1}$ and $\mathrm{O}_{2}$ merge together and saddle-node bifurcation takes place. Then for $\bar{I}>\bar{I}^{*}$, that is in the regions 2 and 3, the only steady state, $\mathrm{O}_{3}$, remains (Fig. 3(b),(c)). It is stable in the region 2 and unstable in the region 3 . The curve $A$, which separates the regions, corresponds to Andronov-Hopf bifurcation. For points on $A$ the state $O_{3}$ changes the stability and in its neighbourghood unstable limit cycle $C^{u}$ appears (above the curve, 


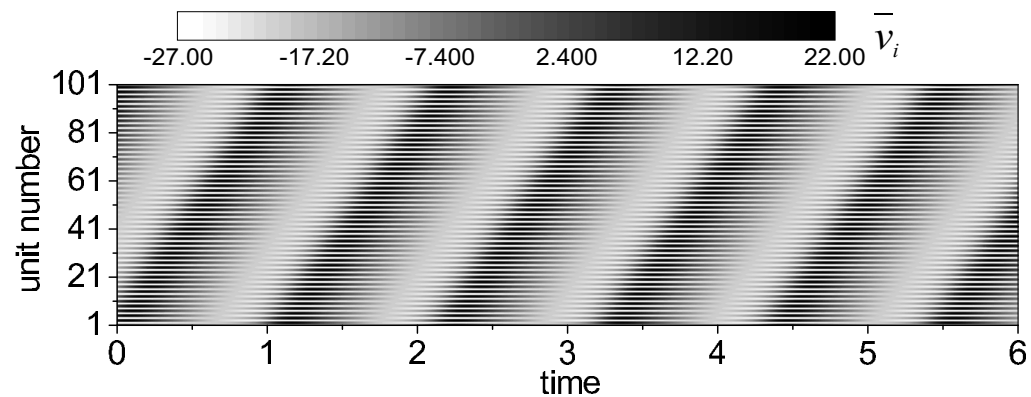

(a)

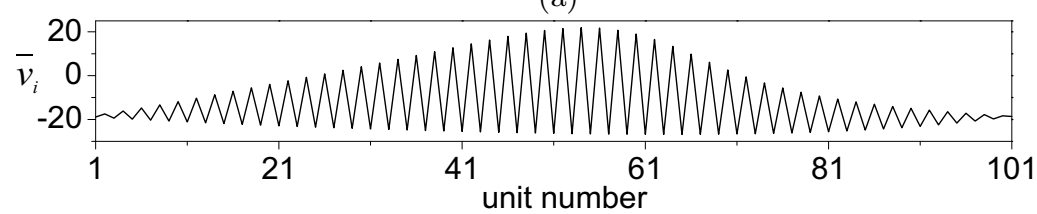

(b)

Fig. 4. Space-time plot (a) and snapshot (b) of typical one-hump anti-phase wave pattern obtained in the ring of 101 units. Parameter values: $\varepsilon=3.28, \bar{I}=48.3, d=0.01$.

see Fig. 3(b)). The curves $H, Q$ and segment of the line $\bar{I}=\bar{I}^{*}$ below the point $P$ correspond to bifurcations that leads to disappearance of the stable limit cycle $C^{s}$. In particular, for points on $Q$ the unstable and stable limit cycles $C^{u}$ and $C^{s}$ merge together and tangent bifurcation takes place. For points on $H$ the limit cycle is glued to the separatrix loop (homoclinic orbit) formed by separatrices $w_{+}^{u}$ and $w_{+}^{s}$ of the saddle $O_{2}$. Finally for points on the line $\bar{I}=\bar{I}^{*}$ below the point $P$ the limit cycle is glued to the homoclinic orbit of saddle-node steady state formed by merging of the states $O_{1}$ and $O_{2}$.

\section{Anti-phase traveling patterns}

Typical wave patterns in neuronal systems include travelling excitation pulses (action potentials), pulse trains (packets of action potentials) and wave fronts (moving interfaces between different levels of neuronal activities). The important property of such waves is that their principal characteristics (profile, velocity) are defined only by intrinsic properties of the medium and do not depend on initial conditions or perturbations within certain limits. But this is the case when the coupling between the elements is strong enough. When the coupling is weak the discrete nature of the system has an effect on its collective behavior. In particular, propagation of pulses, pulse trains and wave fronts becomes impossible (so-called propagation failure phenomenon) due to the existence of a large number of spatially inhomogeneous stationary states in the system [8-11].

Numerical study of the system (1) showed that it yields an interesting kind of localized activity which we called "anti-phase wave patterns". As opposed to aforementioned wave patterns, the latter ones exist even in the case of weak coupling. Figure 4(a) illustrates the evolution of typical anti-phase wave pattern and Fig. 4(b) depicts its snapshot. One can see that the pattern shares some features with envelope solitons found in the nonlinear Schrödinger equation [12,13] and envelope quasisolitons found recently in reaction-cross-diffusion systems [14]. It has the form of spatiotemporal oscillations with a smooth localized envelope which propagates along 

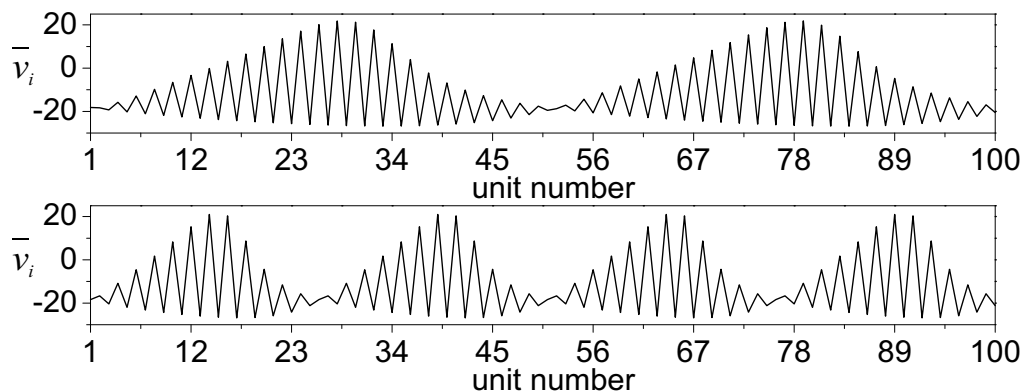

(a)
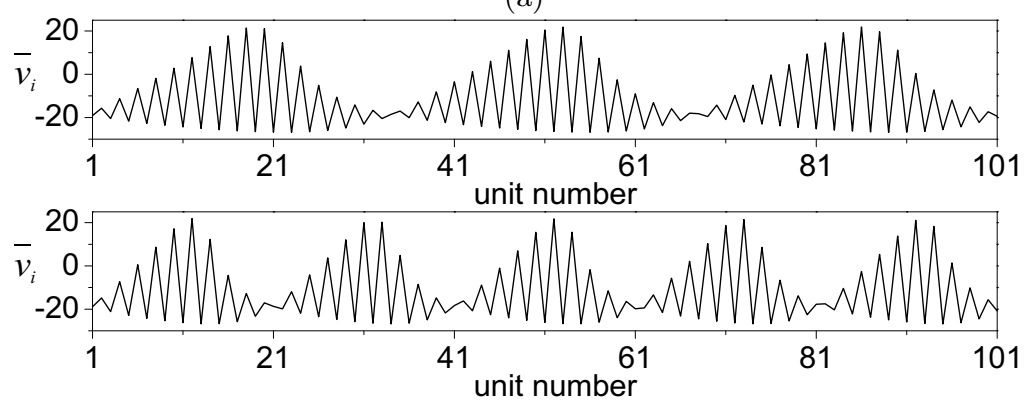

(b)

Fig. 5. Examples of multi-hump anti-phase wave patterns obtained in the rings of (a) 100 and (b) 101 units. Parameter values: $\varepsilon=3.28, \bar{I}=48.3, d=0.01$.

the system preserving its shape and velocity. Note, however, that spatial oscillations due to discreteness are not monotonic but represent instantaneous jumps back and forth between the top and the bottom part of the envelope. It is found that system supports formation of anti-phase wave patterns whose envelope has one (Fig. 4(b)) or more humps (Fig. 5). The patterns with even (odd) number of humps exist in systems with even(odd) number of elements only. Moreover the number of the patterns that exist in a system is proportional to its size. Thus the systems with a large number of elements will show high multistability of the anti-phase wave patterns.

Let us consider the dynamical mechanisms underlying the existence of the antiphase wave patterns. Figure 6 illustrates the projections of phase trajectory of the system (1) corresponding to one-hump anti-phase traveling pattern (Fig. 4) on the planes of membrane potentials of the first two neighboring $\left(\bar{v}_{2}, \bar{v}_{1}\right)$ and the first two next-nearest neurons $\left(\bar{v}_{3}, \bar{v}_{1}\right)$ and evolution of membrane potentials of these neurons. One can see that oscillations of the elements have the same periods. Moreover, oscillations of the neighboring elements are almost anti-phase synchronized, that is the phase shift between them is close (but not equal) to $\pi$, while oscillations of the nextnearest elements are almost complete synchronized (i.e. the phase shift is close to 0 ). The existence of such phase relations is caused by the existence of branches of slow and fast movements [15] on the local limit cycles $C^{s}$ of the elements. By-turn, it can be due to the proximity of the parameters of the elements to the saddle or saddle-node separatrix loop bifurcations, or it can be caused by their relaxational behaviour for $\varepsilon \ll 1$.

The shape of the pattern considered suggests that phase relations analogous to aforementioned ones take place between oscillations of any three neighboring elements. Let us introduce the phase of oscillations of the elements and analyze the phase relations more carefully. Generally speaking, the phase can be rigorously defined for 

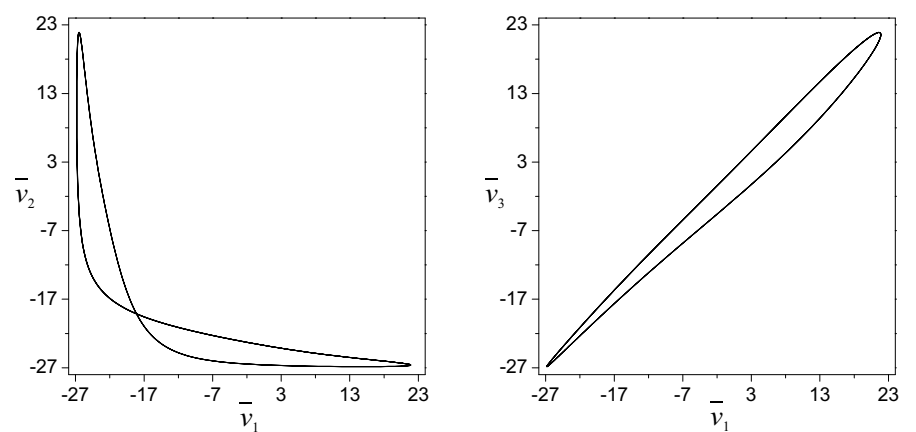

(a)

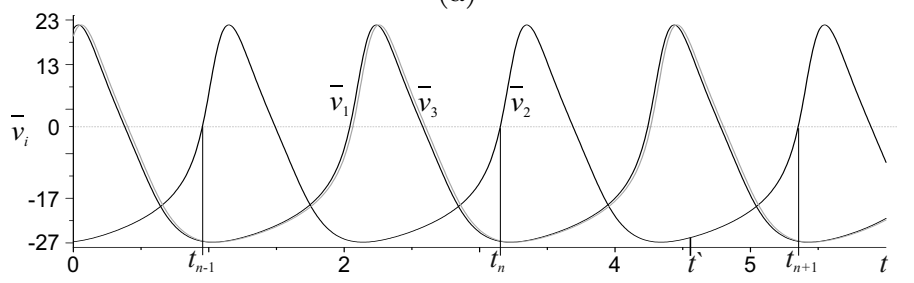

(b)

Fig. 6. The projections of phase trajectory of the system (1) corresponding to the pattern shown in Fig. 4 on the planes of membrane potentials of the first three neighboring neurons (a) and evolution of their membrane potentials (b).

harmonic oscillations only. Still, for nonharmonic periodic or even chaotic oscillations, a quantity that possesses some properties of the classical phase can be introduced [16], and this quantity is also called "the phase". In this paper we define the phase of oscillations as follows. For definiteness, let us consider the $i$-th element. We introduce the sequence $\left\{t_{n}^{i}\right\}$ of instants of the time at which the trajectory of the system crosses the line $\bar{v}_{i}=0$ in the direction corresponding to the increase of variable $\bar{v}_{i}$ (see Fig. 6(b)):

$$
t_{n}^{i}=\left\{t: \bar{v}_{i}(t)=0, \bar{v}_{i}^{\prime}(t)>0\right\} .
$$

Then, the phase of oscillations of the $i$-th element at instant of the time $t^{\prime}$ is determined by the expression

$$
\phi_{i}=2 \pi \frac{t^{\prime}-t_{n}^{i}}{T_{n}^{i}} \text { for } t^{\prime} \in\left[t_{n}^{i}, t_{n+1}^{i}\right]
$$

where $T_{n}^{i}=t_{n}^{i}-t_{n-1}^{i}$ is the "period" of oscillations. Note that such definition of the phase is meaningful only if $T_{n}^{i}$ is constant or sufficiently close to each other. In the former case oscillations of the $i$-th element are regular and the phase increases steadily from 0 to $2 \pi$ all the time. In the latter case oscillations of the element are phasecoherent chaotic and phase piecewise linearly depends on time. Notice that the phase introduced describes the dynamics of an individual element of the system (1) separatelly from the other ones. To describe the dynamics of the system as the whole it is more convenient to consider the quantity: $\varphi_{i}^{j}=\phi_{i}-\phi_{j}$, that is the phase of oscillation of $i$-th element calculated relatively to oscillations of the $j$-th element.

We studied the behaviours of $T_{n}^{i}$ and, for definiteness, $\varphi_{i}^{1}$ for the patterns presented. It is found that in all cases neither $T_{n}^{i}$ nor $\varphi_{i}^{1}$ vary with time and $T_{n}^{i}$ is constant over the entire network. In Fig. 7 the stationary phase distributions corresponding to the patterns are shown. Thus an anti-phase wave pattern represents a certain phase 

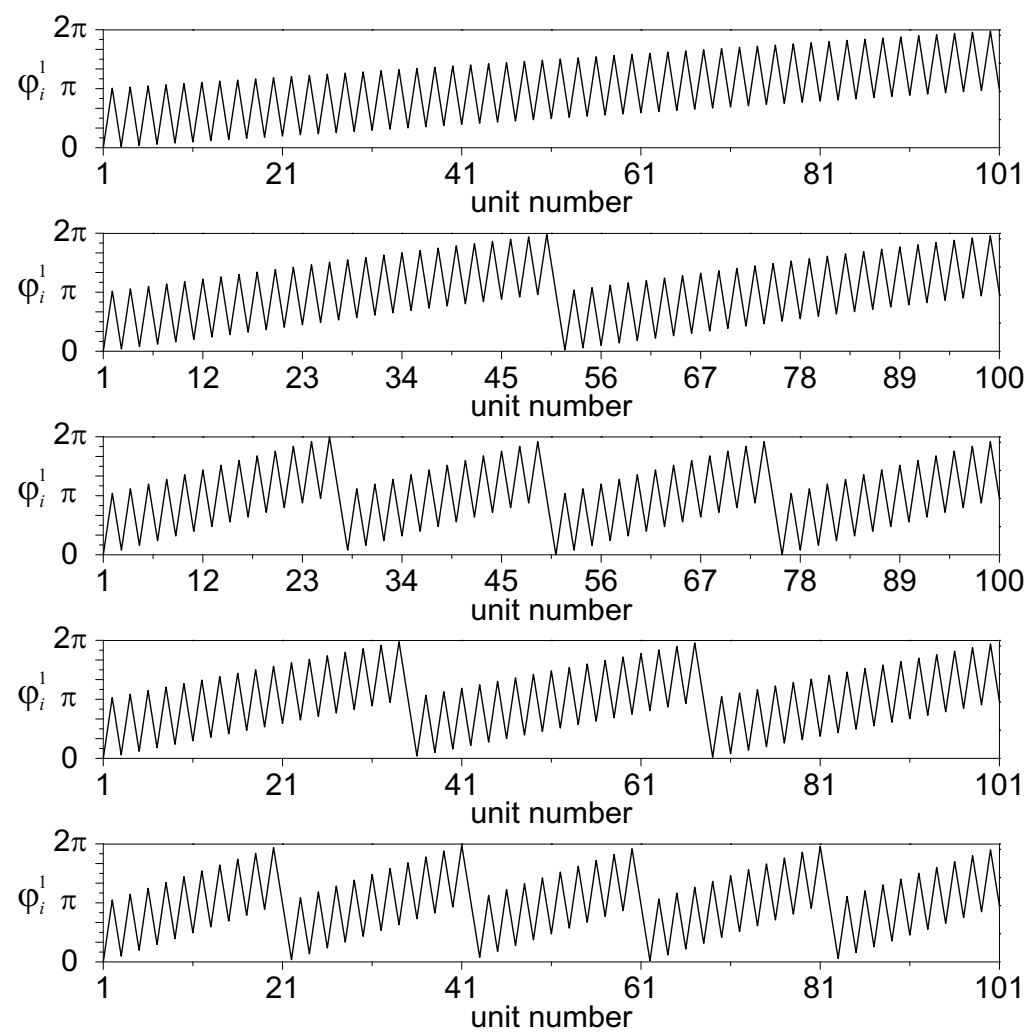

Fig. 7. Phase distributions corresponding to the anti-phase wave patterns shown, respectively, in Figures 4 and 5.

locked solution of the system (1). In this solution each pair of neighboring elements is almost anti-phase synchronized and there is a constant phase shift between pairs that provides the wave nature of the pattern.

Summing up the results of numerical experiments one can obtain the following dependence of the phase on the unit number:

$$
\varphi_{i}^{1}=(\pi+\Delta \varphi)(i-1)
$$

where $\varphi_{i}$ is the phase of oscillation of the $i$-th element, $i=\overline{1, N}$, and $\Delta \varphi$ is a constant phase shift between every pair of elements. Note that since only anti-phase wave patterns with integral number of humps can fit into the full length of the system, the constant phase shift $\Delta \varphi$ can possess only discrete values:

$$
\Delta \varphi=\frac{\pi k}{N}
$$

where $k=0, \pm 1, \pm 2, . .(k \ll N)$ is an anti-phase wave mode number, its absolute value coincides with the number of its humps. Here $k=0$ corresponds to a standing anti-phase wave. The positive values of $k$ correspond to anti-phase wave patterns propagating to the left, while the negative ones correspond to the patterns propagating to the right. Moreover from (8) and (9) it follows that $\varphi_{N+1}^{1}=\pi(N+k)$. It implies that even modes including mode $k=0$ can exist in the system with even number of elements, while odd modes can exist in the system with odd number of elements. Notice that it fully coincides with numerical findings presented. 


\section{The regions of existence of anti-phase wave patterns}

Let us now study the regions of existence of anti-phase wave patterns. Assume that coupling between elements of the system $(1)$ is weak enough $(d \rightarrow 0)$. In this case the theory of weakly coupled oscillators [17-23] can be applied to reduce oscillatory dynamics of the system to the dynamics of a set of first order differential equations describing the evolution of phases of oscillations of its elements. We rewrite the system (1) in the general form:

$$
\frac{d \mathbf{X}_{i}}{d t}=\mathbf{F}\left(\mathbf{X}_{i}\right)+d \mathbf{S}\left(\mathbf{X}_{i}, \mathbf{X}_{i-1}\right)+d \mathbf{S}\left(\mathbf{X}_{i}, \mathbf{X}_{i+1}\right)
$$

where $\mathbf{X}_{i}=\left[\bar{v}_{i}, n_{i}\right]^{T}, \mathbf{F}\left(\mathbf{X}_{i}\right)$ is the local cell vector field defined by right-hand sides of (4) and $\mathbf{S}\left(\mathbf{X}_{j}, \mathbf{X}_{k}\right)=\left[\bar{v}_{k}-\bar{v}_{j}, 0\right]^{T}$.

Recall that, a local element of the system has the stable limit cycle $C^{s}$ (see Fig. 3 ). Let $T$ be a period of the cycle and $\mathbf{G}(t)=\left[\bar{v}^{0}(t), n^{0}(t)\right]^{T}$ a trajectory corresponding to the cycle. Following, Kuramoto [21], we make the transformation of coordinates, $\psi_{i}=\Theta\left(\mathbf{X}_{i}\right)$. Without going into details, notice, that $\Theta(\mathbf{X})$ is an asymptotic phase function, which maps the points from some neighborhood $U$ of the cycle (within its basin of attraction) to a unique phase. And it should satisfy the equation $\frac{d \Theta(\mathbf{X})}{d t}=1$ for all $\mathbf{X} \in U$ when $d=0$. Therefore, if we differentiate $\psi_{i}$ with respect to time using the chain rule for vector functions, we obtain the equation:

$$
\begin{aligned}
\frac{d \psi_{i}}{d t} & =\nabla_{\mathbf{X}} \Theta\left(\mathbf{X}_{i}\right) \frac{d \mathbf{X}_{i}}{d t}= \\
& =\nabla_{\mathbf{X}} \Theta\left(\mathbf{X}_{i}\right) \mathbf{F}\left(\mathbf{X}_{i}\right)+d \nabla_{\mathbf{X}} \Theta\left(\mathbf{X}_{i}\right)\left[\mathbf{S}\left(\mathbf{X}_{i}, \mathbf{X}_{i-1}\right)+\mathbf{S}\left(\mathbf{X}_{i}, \mathbf{X}_{i+1}\right)\right]= \\
& =1+d \nabla_{\mathbf{X}} \Theta\left(\mathbf{X}_{i}\right)\left[\mathbf{S}\left(\mathbf{X}_{i}, \mathbf{X}_{i-1}\right)+\mathbf{S}\left(\mathbf{X}_{i}, \mathbf{X}_{i+1}\right)\right]
\end{aligned}
$$

One can see that the equation (11) is exact but it is not closed with respect to the phase variables $\psi_{i}$. Hoverer, since $d$ is weak, then $\mathbf{X}_{i}(t)$ is close to $\mathbf{G}(t)$, which is close to $\mathbf{G}\left(\psi_{i}\right)$. Thus (11) becomes:

$$
\frac{d \psi_{i}}{d t}=1+d \nabla_{\mathbf{X}} \Theta\left(\mathbf{G}\left(\psi_{i}\right)\right)\left[\mathbf{S}\left(\mathbf{G}\left(\psi_{i}\right), \mathbf{G}\left(\psi_{i-1}\right)\right)+\mathbf{S}\left(\mathbf{G}\left(\psi_{i}\right), \mathbf{G}\left(\psi_{i+1}\right)\right)\right] .
$$

By introducing the variables, $\phi_{i}=-t+\psi_{i}$, we have:

$$
\begin{aligned}
\frac{d \phi_{i}}{d t}=d \nabla_{\mathbf{X}} \Theta\left(\mathbf{G}\left(t+\phi_{i}\right)\right)\left[\mathbf { S } \left(\mathbf{G}\left(t+\phi_{i}\right)\right.\right. & \left., \mathbf{G}\left(t+\phi_{i-1}\right)\right)+ \\
& \left.+\mathbf{S}\left(\mathbf{G}\left(t+\phi_{i}\right), \mathbf{G}\left(t+\phi_{i+1}\right)\right)\right]
\end{aligned}
$$

Note that $\phi_{i}$ evolves on a much slower timescale than $T$. Since the functions in the equation (12) are smooth and $\mathbf{G}(t)$ is $T$-periodic we can eliminate the explicit time dependence from its right-hand side by "averaging" over the period $T[24,25]$. Then, by exploiting the fact, that the second component of coupling function $\mathbf{S}$ is zero, we arrive at:

$$
\frac{d \phi_{i}}{d t}=d\left[H\left(\phi_{i-1}-\phi_{i}\right)+H\left(\phi_{i+1}-\phi_{i}\right)\right]
$$

where

$$
H(\phi)=\frac{1}{T} \int_{0}^{T} \frac{\partial \Theta(\mathbf{G}(t))}{\partial \bar{v}}\left[\bar{v}^{0}(t+\phi)-\bar{v}^{0}(t)\right] d t, H(\phi)=H(\phi+T) .
$$

Finally to complete the reduction we must compute the partial derivative of the asymptotic phase function $\Theta(\mathbf{X})$ with respect to $\bar{v}$. Unfortunately, in practice the straightforward calculation of $\Theta(\mathbf{X})$ for arbitrary $\mathbf{X}$ is not a trivial task. However, there are several ways of direct computation of the partial derivatives of $\Theta(\mathbf{X})$ evaluated at the limit cycle solution $\mathbf{G}(t)$ which have shown to be very efficient. Here 
we use the adjoint method based on the results of application of the singular perturbation approach to a weakly coupled oscillators [17-20]. They state that the partial derivatives of $\Theta(\mathbf{G}(t))$ are components of $T$-period solution of the adjoint equation for the local cell linearized around the limit cycle:

$$
\frac{d \mathbf{Z}}{d t}=-D \mathbf{F}(\mathbf{G}(t))^{T} \mathbf{Z}
$$

subject to the normalization constraint

$$
\mathbf{Z}(0) \mathbf{G}^{\prime}(0)=1 .
$$

In practice, the solution to (15) is found by integrating the equation backward in time [26] since the system has the opposite stability of the original system.

In previous section we showed that an anti-phase wave pattern represents phase locked solution of the system (1) with the form:

$$
\begin{aligned}
& \phi_{i}(t)=\Omega t+\varphi_{i}, \\
& \varphi_{i}-\varphi_{i-1}=\pi+\Delta \varphi .
\end{aligned}
$$

By substituting (16) into (13) one can obtain the following relation:

$$
\Omega=\bar{H}(-0.5-\sigma)+\bar{H}(0.5+\sigma) .
$$

where $\sigma=\frac{k}{2 N}$ is a wave number and $\bar{H}(\phi)=H(T \phi)$. In fact the equation (17) defines the dependence of an anti-phase wave pattern local oscillations frequency $\Omega$ on its wave number $\sigma$. The example of relation $\Omega(\sigma)$ and the functions it depends on calculated for $\varepsilon=3.28, \bar{I}=48.3$ are shown in Fig. 8 .

Let us now analyze the linear stability of phase locked solution (16). We suppose that $\varphi_{i}$ are not constant but depend on the time and introduce the variables, $\rho_{i}(t)=$ $\phi_{i}(t)-\phi_{i-1}(t)$. It can be readily shown that the dynamics of $\rho_{i}$ satisfies:

$$
\frac{d \rho_{i}}{d t}=d\left[-H\left(-\rho_{i-1}\right)+H\left(-\rho_{i}\right)-H\left(\rho_{i}\right)+H\left(\rho_{i+1}\right)\right] .
$$

Note that anti-phase wave patterns correspond to spatially-homogeneous stationary states of equation (18). Thus by analyzing stability of the stationary states of (18) one can know stability of anti-phase wave patterns. Linearizing (18) about the state $\rho_{i}=\rho^{0}=0.5+\sigma$ we have

$$
\frac{d \xi_{i}}{d t}=d\left[a \xi_{i-1}-(a+b) \xi_{i-1}+b \xi_{i+1}\right]
$$

where $\xi_{i}=\rho_{i}-\rho^{0}$ are infinitesimal deviations from the stationary state and $a=$ $\bar{H}^{\prime}(-0.5-\sigma), b=\bar{H}^{\prime}(0.5+\sigma)$. Then, taking into account the periodic boundary conditions, we can represent $\xi_{i}$ as discrete Fourier series [8] and derive the spectra of Lyapunov characteristic eigenvalues associated with the stationary state $\rho^{0}$ :

$$
\lambda_{i}=-(b+a)+(b+a) \cos \left[(i-1) \frac{2 \pi}{N}\right]+\mathcal{I}(b-a) \sin \left[(i-1) \frac{2 \pi}{N}\right],
$$

where $\mathcal{I}$ is the imaginary unit. By analyzing the spectra (20) one can obtain the following stability condition for spatially-homogeneous stationary states of (18) and so for anti-phase wave patterns:

$$
\bar{H}^{\prime}(-0.5-\sigma)+\bar{H}^{\prime}(0.5+\sigma)>0 .
$$

The fig. 9(a) illustrates in $(\varepsilon, \bar{I})$-plane the region of existence of stable anti-phase wave patterns with wave numbers belonging in the interval $\sigma \in[0,0.05]$. We see that for 

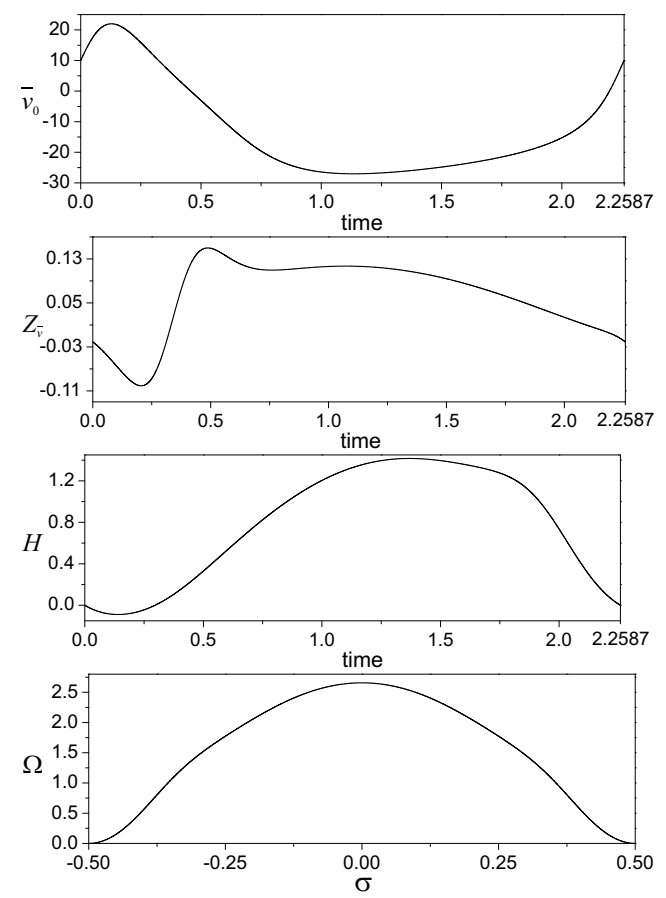

Fig. 8. The images from top to bottom are waveforms of membrane potential $\bar{v}_{0}$ corresponding to the local limit cycle $C^{s}, \bar{v}$-component $Z_{\bar{v}}$ of a solution to the adjoint problem (15), interaction function $H$ defined by (14), and the relation $\Omega(\sigma)$ for solutions of the form (16), respectively. Parameter values: $\varepsilon=3.28, \bar{I}=48.3$.

the values of parameter $\bar{I}<\bar{I}^{*}$ (see section 2.1) the region coincides with the domain of existence of oscillatory behaviour in a local cell. On the other hand, for $\bar{I}>\bar{I}^{*}$ the region is localized in neighborhood of upper boundary of oscillatory cell behaviour, that is curve $Q$. Though the boundary of the region of existence is sharp enough its structure is by no means trivial. Indeed, in Fig. 9(b),(c) two typical pictures of the structure of the boundary far to the left and to the right from the point $P$, respectively, are shown. If we cross the boundary to the left of $P$ (Fig. 9(b)) then the smaller an anti-phase a wave pattern has wave number the earlier the pattern becomes unstable. On the other hand if we cross the boundary to the right of $P$ (Fig. 9(c)) then the larger the pattern has a wave number the earlier it becomes unstable. Note that the point $P$ is a point of intersection of the boundaries of existence regions of anti-phase wave patterns with $\sigma=0$ and $\sigma=0.05$. In its neighborhood the structure of the boundary is even more complex. Here the boundary of existence of each anti-phase wave pattern intersects the boundaries of other anti-phase wave patterns with smaller wave numbers forming fractal-like parameters set.

\section{Conclusions}

Spatio-temporal dynamics of a ring system modeling collective behavior of electrically coupled oscillatory neurons have been investigated. It has been found that for small values of coupling strength, when the discreteness affects its collective behavior, the system is capable to produce a special class of localized patterns so called "anti-phase 


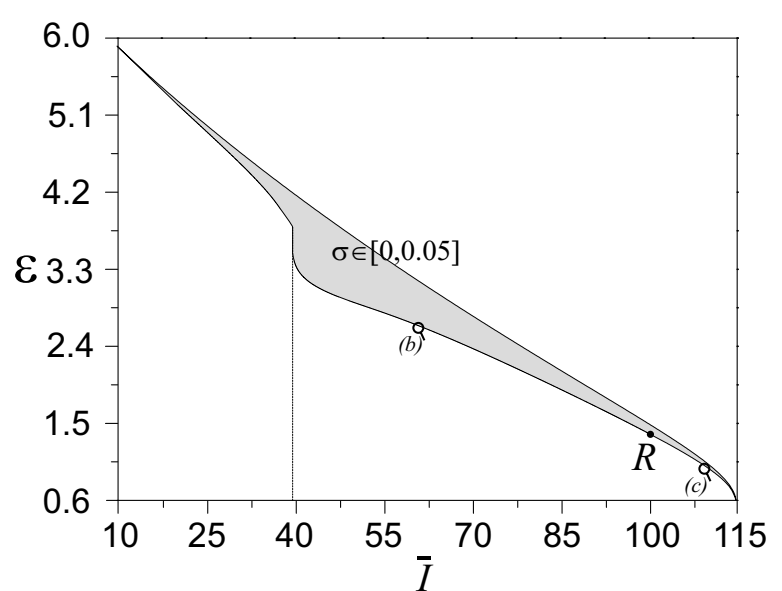

(a)

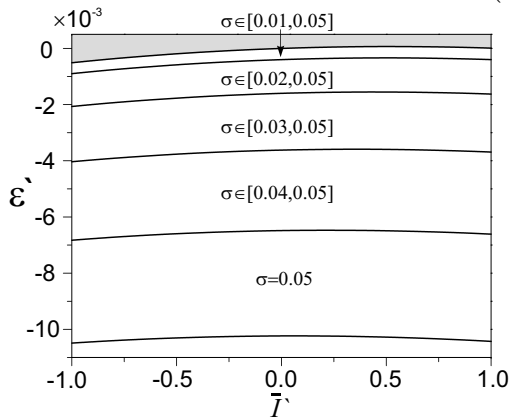

(b)

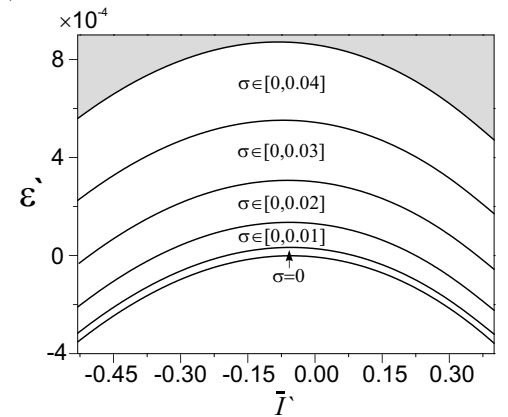

(c)

Fig. 9. The region (a) of existence of anti-phase wave patterns with wave numbers $\sigma \in$ $[0,0.05]$ on $(\varepsilon, \bar{I})$-plane and enlarged fragments (a), (b) of its bottom boundary near the points $(2.6661,60.09),(0.9774,110.06)$, respectively, in coordinate system $\left(\varepsilon^{\prime}, \bar{I}^{\prime}\right)$ rotated tangent to the boundary. The point $R(1.4233,98.88)$ is a point of intersection of the bottom boundaries of existence regions of anti-phase wave patterns with $\sigma=0$ and $\sigma=0.05$.

wave patterns". The patterns look like the envelope solitons. Indeed they have the form of spatiotemporal oscillations with a smooth localized envelope which propagates along the system preserving its shape and velocity. However, due to discreteness, spatiotemporal oscillations represent instantaneous jumps back and forth between the top and the bottom part of the envelope. It has been shown that the patterns with different number of humps can be formed under the same values of parameters, indicating high multistability. Moreover the patterns with even number of humps can be formed only in the system with even number of elements, while the patterns with odd number of humps exist only in the system with odd number of elements.

The mechanisms of the formation of anti-phase wave patterns has been discussed. In particular, it has been shown that formation of the patterns is related to establishing of a certain phase relations between oscillations of neighboring elements. Indeed each pair of neighboring elements in the system is almost anti-phase synchronized and there is a constant phase shift between pairs that provides the wave nature of the pattern.

By using the weakly coupled oscillators theory the regions of the existence of anti-phase wave patterns are studied. It has been shown that in the domain where a 


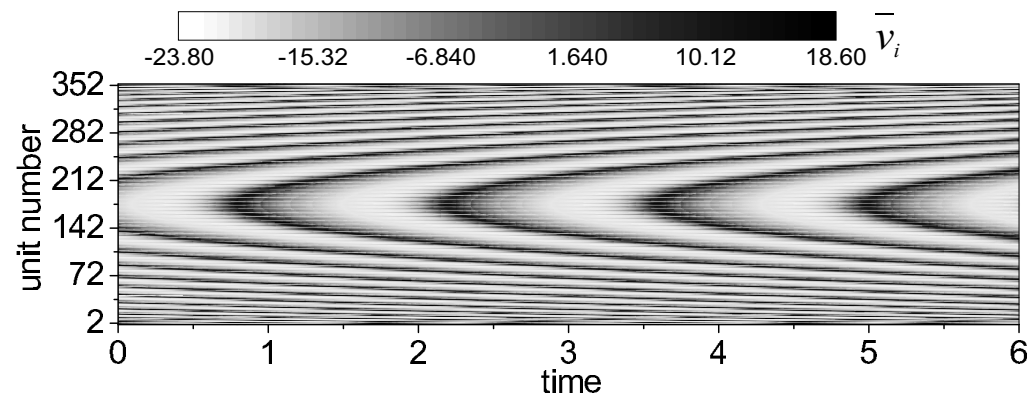

(a)

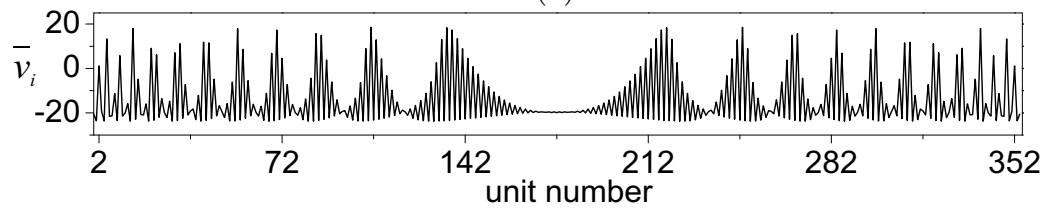

(b)

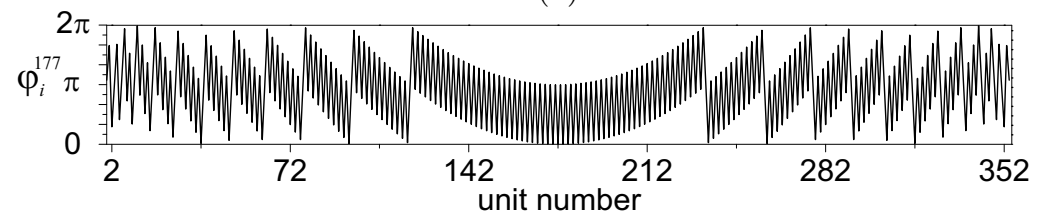

(c)

Fig. 10. Space-time plot (a), snapshot (b) and corresponding phase distribution (c) of typical anti-phase wave pattern obtained in the network of 355 units with non-periodic (zero flux) boundaries. Parameter values: $\varepsilon=4, \bar{I}=38.2, d=0.01$.

cell has three stationary states the region of existence of patterns coincides with the domain of oscillatory cell behaviour, if not it is localized in a neighborhood of upper boundary of oscillatory cell behaviour. More over it is found that its boundary has a complex, even fractal-like, structure.

Note that the anti-phase wave patterns can exist also in systems with non-ring structure. For example, Figure 10 illustrates the typical anti-phase wave pattern found in a chain of locally coupled neural-like units (4) with zero flux boundaries.

This work has been supported by the Russian Foundation for Basic Research (Grants 12-02-00526, 12-02-31825, 13-02-00858) and the Federal Target Program "Scientific and scientific-pedagogical personnel of the innovative Russia" in 2009-2013 (Contracts 8497, 8205).

\section{References}

1. G. B. Ermentrout and D. H. Terman, Mathematical Foundations of Neuroscience (Springer, New York, 2010).

2. E. Kaplan and E. Benardete, Progress in Brain Research 134 (2001) 17-34.

3. M. J. Berry, D. K. Warland and M. Meister, Neural Network 14 (2001) 611-615.

4. G. D. Field and E. J. Chichilnisky, Information Processing in the Primate Retina: Circuitry and Coding (Annual Review of Neuroscience, 2007).

5. E.R. Kandel, J.H. Schwartz, T.M. Jessell, Principles of neural science (Prentice-Hall Intern. Inc., New Jersey, 1991).

6. C. Morris and H. Lecar, Biophys. J. 35, (1981) 193-213. 
7. K. Tsumoto, H. Kitajima T. Yoshinaga, K. Aihara, H. Kawakami, Neurocomputing 69 (2006) 293-316.

8. V.I. Nekorkin, M.G. Velarde, Sinergetic phenomena in active lattices (Springer, Berlin, 2002).

9. J.P. Keener, SIAM Journal on Applied Mathematics 47 (1987) 556-572.

10. J.C. Comte, S. Morfu, P. Marquie, Physical Review E 64 (2001) 027102-1-4.

11. S. Binczak, J.M. Bilbault, Int. J. Bifurcation and Chaos 14 (2004) 1819-1830.

12. M.C. Cross, P. C. Hohenberg, Rev. Mod. Phys. 65 (1993) 851-1112.

13. B. Malomed, in Encyclopedia of Nonlinear Science, edited by A. Scott (Routledge, New York, 2005) 639-642.

14. V.N. Biktashev, M.A. Tsyganov, Physical Review Letters 107 (2011) 134101-1-4.

15. D.E. Postnov, S.K. Han, Technical Physics Letters 25 (1999) 128-130.

16. V.S. Anishchenko, V. Astakhov, A. Neiman, T. Vadivasova, L. Schimansky-Geier, Nonlinear Dynamics of Chaotic and Stochastic Systems (Springer, Berlin, 2007).

17. I. Malkin, Metodi Puankare $i$ Liapunova $v$ teorii nelineinix kolebanii (Gostexizdat, Moscow, 1949).

18. I. Malkin, Nekotorye zadachi teorii nelineinix kolebanii (Gostexizdat, Moscow, 1956).

19. J. Neu, SIAM J. Appl. Math. 37 (1979) 307-315.

20. G. Ermentrout, N. Kopell, SIAM J. Math. Anal. 15 (1984) 215-237.

21. Y. Kuramoto, Chemical Oscillations, Waves, and Turbulence (Springer, New York, 1984).

22. F.C. Hoppensteadt, E.M. Izhikevich, Weakly Connected Neural Networks (Springer, New York, 1997).

23. T. Lewis, J. Rinzel, Comp. Neurosci. 14 (2003) 283-309.

24. J. Guckenheimer, P. Holmes, Nonlinear Oscillations, Dynamical Systems, and Bifurcations of Vector Fields (Springer,New York, 1983).

25. G. Ermentrout, N. Kopell, J. Math. Bio. 29 (1991) 33-44.

26. T. Williams, G. Bowtell, J. Comput. Neurosci. 4 (1997) 47-55. 